\title{
Expression of YAP/TAZ in Keratocystic Odontogenic Tumors and Its Possible Association with Proliferative Behavior
}

\author{
Qi-Wen Man, ${ }^{1}$ Yan-Qi Ma, ${ }^{1}$ Jin-Yuan Liu, ${ }^{1}$ Yi Zhao, ${ }^{2}$ Bing Liu, ${ }^{3}$ and Yi-Fang Zhao ${ }^{3}$ \\ ${ }^{1}$ The State Key Laboratory Breeding Base of Basic Science of Stomatology and Key Laboratory of \\ Oral Biomedicine Ministry of Education, School and Hospital of Stomatology, Wuhan University, Wuhan, China \\ ${ }^{2}$ Department of Prosthodontics, School and Hospital of Stomatology, Wuhan University, Wuhan, China \\ ${ }^{3}$ Department of Oral and Maxillofacial Surgery, School and Hospital of Stomatology, Wuhan University, Wuhan, China \\ Correspondence should be addressed to Bing Liu; liubing9909@sina.cn and Yi-Fang Zhao; yifang@whu.edu.cn
}

Received 6 November 2016; Accepted 20 March 2017; Published 23 April 2017

Academic Editor: Satoshi Maruyama

Copyright (C) 2017 Qi-Wen Man et al. This is an open access article distributed under the Creative Commons Attribution License, which permits unrestricted use, distribution, and reproduction in any medium, provided the original work is properly cited.

\begin{abstract}
The aim of this study is to clarify whether YAP/TAZ is involved in the pathogenesis and proliferative growth of keratocystic odontogenic tumor (KCOT). The expression levels of YAP/TAZ and downstream proteins and genes in normal oral mucosa (OM) and KCOT were determined and compared by immunohistochemistry and real-time quantitative PCR. The results showed that the expression of YAP/TAZ and downstream proteins (Cyr61, CTGF) was significantly upregulated in KCOT with upregulation of Ki-67 compared to OM. Importantly, the mRNA levels of transcription factors (TEAD1, TEAD4, and RUNX2) and cell cycle related genes (CDK2, PCNA), which interact with the transcriptional coactivators YAP/TAZ, are also upregulated in the KCOT. In addition, the results from Spearman rank correlation test revealed the close relationship between YAP/TAZ and Ki-67, which was further evidenced by double-labelling immunofluorescence that revealed a synchronous distribution for YAP/TAZ with Ki-67 in KCOT samples. All the data suggested YAP/TAZ might be involved in the proliferative behavior of KCOT.
\end{abstract}

\section{Introduction}

Keratocystic odontogenic tumor (KCOT) is formerly known as odontogenic keratocyst (OKC) [1]. In 2005, OKC was reclassified as a benign neoplasm of odontogenic origin under the name of "keratocystic odontogenic tumor" by the World Health Organization [2]. In the cysts and tumors of jaw bones, KCOT has been reported as the second most common in the Chinese population [3]. The KCOT has some particular histologic features compared with other odontogenic lesions: parakeratinized stratified cell layers, daughter cysts, budding proliferation of the epithelium, and the formation of the islands of odontogenic epithelium [4]. Quite unexpectedly, the KCOT has a great potential to recur and the recurrence rate ranged from 0 to 100 percent due to different observation periods and treatments [5]. It has been shown that the expressions of cellular proliferative markers (Ki-67, PCNA, and Cyclin D1) of the epithelium were consistent with the aggressive features of the KCOT $[6,7]$.
Recently, the aberrant activation of the transcriptional regulators YAP/TAZ was proved to contribute to the initiation and progression of a range of tumors [8]. YAP/TAZ transcriptional activity is dependent on their recruitment to the nucleus, which promotes binding to a range of transcription factors, most notably the TEAD family [9]. The transcription complexes regulate expression of a number of downstream target genes such as CTGF and Cyr61 [8, 9]. YAP/TAZ-directed transcription promotes cell proliferation, prosurvival, and cell migration signals, all of which contribute to the protumorigenic roles of YAP/TAZ [8]. Multiple signaling events restrict YAP/TAZ from the nucleus, the best characterized of which are signals mediated by the Hippo pathway [9]. Besides, the mechanical cues and signals that affect cytoskeletal dynamics, such as those signals transduced by G-protein coupled receptors, also control YAP/TAZ localization [10]. In the oral and maxillofacial regions, dysregulated YAP/TAZ activity has been reported to be implicated in head and neck tumors [11]. However, 
the expression of YAP/TAZ and its downstream proteins in KCOT is still not clear.

In the present study, therefore, we detected the expression of YAP/TAZ, Cyr61, CTGF, Ki-67, YAP, and TAZ in clinical samples of KCOT and OM using immunohistochemical, immunofluorescence staining, and western blot analysis, respectively. The transcription factors (TEAD1, TEAD4, and RUNX2), cell cycle related genes (CDK2, PCNA), YAP, and TAZ genes were compared using RT-qPCR analysis. The correlation between the nucleus expression level YAP/TAZ and the cell proliferative marker (Ki-67) in KCOT was also explored using Spearman rank correlation and double immunofluorescence staining. This is the first study that has evaluated the dysregulated status of YAP/TAZ transcriptional activity in KCOT and explored its possible association with the growth potential.

\section{Materials and Methods}

2.1. Specimens. Twenty-one samples of human KCOT and eight samples of human oral mucosa (OM) were collected after operations at the Hospital of Stomatology, Wuhan University. The procedures were implemented according to the National Institutes of Health guidelines regarding the use of human tissues. The study was approved by the review board of the Ethics Committee of Hospital of Stomatology, Wuhan University. The pathological diagnosis of KCOT was confirmed by haematoxylin and eosin staining based on the criteria described by the WHO classification. All samples were fixed immediately in $4 \%$ buffered paraformaldehyde and routinely embedded in paraffin; then sections approximately $4 \mu \mathrm{m}$ thick were cut from the paraffin blocks for immunostaining.

2.2. Immunohistochemistry. Immunostaining was carried out with the streptavidin biotin method on dewaxed tissue sections as previously described [12]. Briefly, $4 \mu \mathrm{m}$ thick sections were processed for subsequent histological and immunohistochemical study. Primary antibodies were YAP/TAZ (Cell Signaling Technology, rabbit, 1:50, \#8418), Cyr61 (Proteintech, rabbit, $1: 200$, \#26689-1-AP), CTGF (Proteintech, rabbit, 1:200, \#23936-1-AP), Ki-67 (Abcam, mouse, 1: 200, \#ab8191), YAP (Cell Signaling Technology, rabbit, 1:100,\#14074), and TAZ (Cell Signaling Technology, rabbit, 1:100, \#4883). To visualize the reaction, 3,30-diaminobenzidine (DAB Chromogen, DakoCytomation) was used. Finally, slides were counterstained by Mayer's hematoxylin for 10 seconds. A brown colored cytoplasmic reaction was considered to indicate immunopositivity for Cyr61 and CTGF. Overlapping nuclei sections were considered positive immunopositivity for YAP/TAZ, Ki-67, YAP, and TAZ. For quantization, the numbers of stained cells were counted at an original magnification 200x in 5 random fields by 2 investigators in the cyst wall layer regions. For YAP/TAZ, Ki-67, YAP, and TAZ quantization, the immunoscore was expressed as the percentage of stained cells. For Cyr61 and CTGF evaluation, the staining scores were evaluated as the sums of staining intensity $(0=$ no staining; $1=$ mild staining; $2=$ moderate staining; and $3=$ intense staining) and the percentage of stained cells $(0 \leq 10 \% ; 1=11-25 \% ; 2=26-50 \% ; 3=51-75 \%$; and $4>75 \%$ of the KCOT and OM epithelial cells).

2.3. Western Blotting. Proteins were extracted from RNA later-preserved tissues from KCOT and OM. The tissue homogenate was prepared to disrupt the cell membranes under precooling conditions. After centrifugation at the speed of $14,000 \mathrm{~g}$ for 10 minutes, the protein containing supernatant was collected and the concentration of the protein was estimated using the BCA assay (Pierce, Rockford, IL, USA). Supernatants of the KCOT and OM collections were boiled for 5 minutes in SDS gel loading buffer before electrophoresis. $20 \mu \mathrm{g}$ of protein was separated on $10 \%$ SDS-polyacrylamide gels and transferred onto polyvinylidene fluoride (PVDF) membranes (Roche Applied Science). The blots were blocked overnight with $5 \%$ nonfat dry milk and incubated with primary antibody at dilutions YAP (Cell Signaling Technology, rabbit, 1:1000, \#14074), TAZ (Cell Signaling Technology, rabbit, $1: 2000$, \#4883), and GAPDH (Santa Cruz Biotechnology, mouse, $1: 1000$, \#sc-32233). Immunoblots were detected by HRP-conjugated secondary antibodies (Pierce) using the chemiluminescence kit (Pierce). The proteins were detected by the ECL detection system and the light emitted was captured on film.

2.4. Real-Time Quantitative Polymerase Chain Reaction (RT$q P C R)$. Total RNA was isolated from tissues of four cases of KCOT and three cases of OM by using an RNeasy Mini kit (Qiagen, Hilden, Germany) according to the manufacturer's instructions. The RNA $(2 \mu \mathrm{g})$ was transcribed into cDNA using the PrimeScript 1st Strand cDNA Synthesis Kit (Takara, Otsu, Japan). The transcribed product was amplified by polymerase chain reaction (PCR) into a final volume of $20 \mu \mathrm{L}$ by using the following sense and antisense primers: TEAD1: $5^{\prime}$ ATGGAAAGGATGAGTGACTCTGC- $3^{\prime}$ and $5^{\prime}$-TCCCACATGGTGGATAGATAGC-3'; TEAD4: $5^{\prime}$-GAACGGGGACCCTCCAATG-3' $3^{\prime}$ and $5^{\prime}$-GCGAGCATACTCTGTCTCAAC-3'; RUNX2: $5^{\prime}$-TGGTTACTGTCATGGCGGGTA-3' and $5^{\prime}$-TCTCAGATCGTTGAACCTTGCTA-3'; CDK2: $5^{\prime}$ CCAGGAGTTACTTCTATGCCTGA- $3^{\prime}$ and $5^{\prime}$-TTCATCCAGGGGAGGTACAAC-3'; PCNA: $5^{\prime}$-CCTGCTGGGATATTAGCTCCA-3' and $5^{\prime}$-CAGCGGTAGGTGTCGAAGC-3'; YAP: $5^{\prime}$-ACGTTCATCTGGGACAGCAT-3' and $5^{\prime}$-GTTGGGAGATGGCAAAGACA-3'; TAZ: $5^{\prime}$-ATTCATCGCCTTCCTAGGGT- $3^{\prime}$ and reverse $5^{\prime}$-GGCTGGGAGATGACCTTCAC-3'; GAPDH: $5^{\prime}$-CCATGTTCGTCATGGGTGTGAACCA- $3^{\prime}$ and $5^{\prime}$-GCCAGTAGAGGCAGGGATGATGTTC-3'. GAPDH was selected as the internal control for each experiment.

2.5. Double-Labelling Immunofluorescence. Double-labelling immunofluorescence was done as described previously [12]. Briefly, $4 \mu \mathrm{m}$ thick sections were processed for subsequent immunofluorescence staining. The primary antibodies for double-labelling immunofluorescence were YAP/TAZ (Cell Signaling Technology, rabbit, 1:50, \#8418), Cyr61 (Proteintech, rabbit, 1:100, \#26689-1-AP), CTGF (Proteintech, 

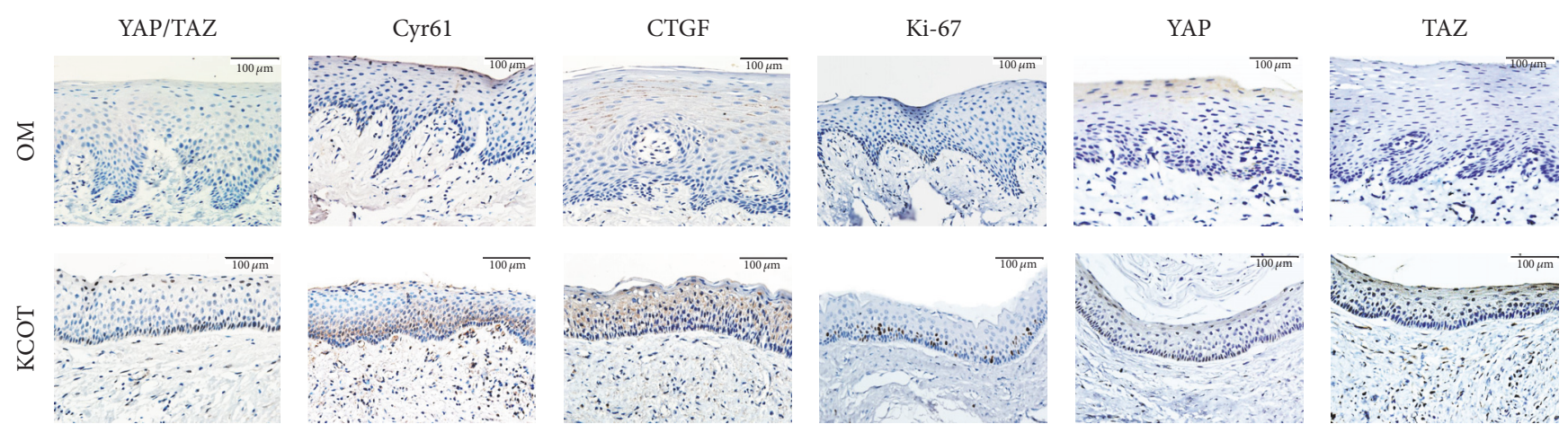

(a)

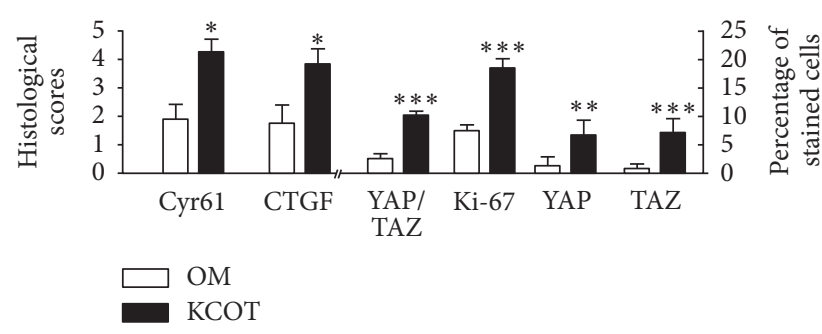

(b)

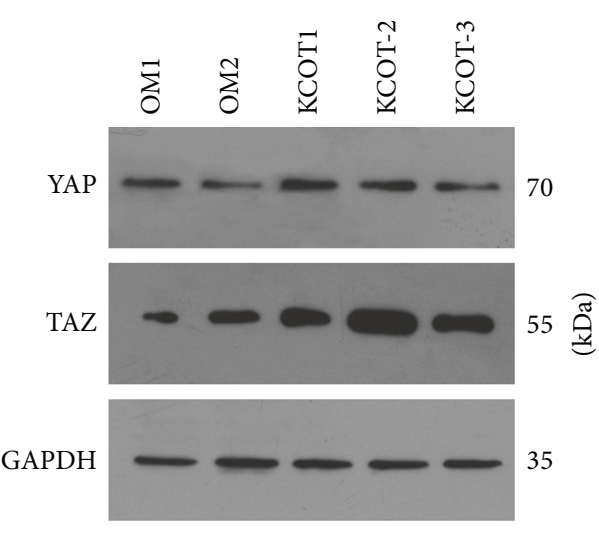

(c)

Figure 1: (a) YAP/TAZ, Cyr61, CTGF, Ki-67, YAP, and TAZ expression in normal oral mucosa and keratocystic odontogenic tumors. (b) Immunostaining scores for indicated markers in samples from both normal oral mucosa and keratocystic odontogenic tumors. All values are mean (SEM), ${ }^{*} p<0.05,{ }^{* *} p<0.01$, and ${ }^{* * *} p<0.001$. (c) Western blotting for YAP, TAZ, and GAPDH in the samples from oral mucosa and keratocystic odontogenic tumor.

rabbit, 1:100, \#23936-1-AP), and Ki-67 (Abcam, mouse, $1: 100$, \#ab8191). Double-labelling immunofluorescence for YAP/TAZ and DAPI, YAP/TAZ and Ki-67, and Cyr61 and CTGF was performed in samples of both KCOT and OM. The nuclei were counterstained with 40,6-diamidino-2-phenylindole (DAPI) for 10 minutes at room temperature. The slides were stored in $-20^{\circ} \mathrm{C}$ for later detection.

\section{Results}

3.1. Upregulated Expression of YAP/TAZ, Cyr61, CTGF, Ki-67, YAP, and TAZ Proteins in KCOT. To investigate the activation status of YAP/TAZ in KCOT, the expression of the YAP/TAZ, and the downstream proteins Cyr61, CTGF was explored and compared in the twenty-one KCOT samples and eight normal oral mucosa samples by immunochemistry. In the sections of KCOT, the tested proteins including YAP/TAZ, CTGF, Cyr61, Ki-67, YAP, and TAZ were expressed in the epithelial layers (Figure 1(a)). Specifically, the YAP/TAZ and Ki-67 were mainly located in the nucleus of the basal cell layers of KCOT; YAP and TAZ were located in the nucleus of the basal and superficial epithelial layers of KCOT. Additionally, TAZ proteins were also frequently expressed within the stromal component of KCOTs. Also, the immunofluorescence staining of the YAP/TAZ in KCOT showed overlapping with DAPI indicating the nucleus expression. However, few overlaps of YAP/TAZ and DAPI were observed in the OM (see Supplementary Figure 1 in Supplementary Material available online at https://doi.org/10.1155/2017/4624890, white arrow). The Cyr61 and CTGF were mainly localized in the cytoplasm of the epithelium of KCOT. In contrast, the immunoreactivities of the YAP/TAZ and downstream proteins (Cyr61, CTGF) in KCOT showed more expression compared with these from OM. More importantly, the data further confirmed that the expressions of YAP/TAZ $(p<0.0001)$, Cyr61 $(p=0.0103)$, CTGF $(p=0.0467), \operatorname{Ki}-67(p=0.0006)$, YAP $(p=0.0073)$, and TAZ $(p<0.0001)$ were significantly greater in samples of KCOT than those from OM (Figure 1(b)).

3.2. Western Blot Analysis. To confirm the presence of YAP and TAZ proteins in the KCOT and OM, western blotting of YAP and TAZ was performed using the tissue lysates from two cases of OM and three cases of KCOTs. The results showed the bands of the expected sizes of $70 \mathrm{kDa}$ (YAP) and $55 \mathrm{kDa}$ (TAZ) for all the analysed samples. The band for $55 \mathrm{kDa}$ (TAZ) protein in OM was weaker than that in KCOT samples (Figure 1(c)). 


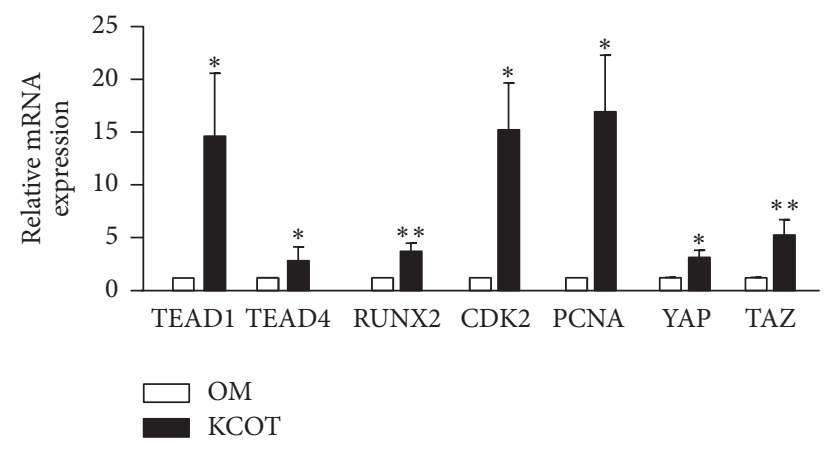

FIgURE 2: The mRNA expression levels of TEAD1, TEAD4, RUNX2, CDK2, PCNA, YAP, and TAZ in samples from both keratocystic odontogenic tumors (solid bars) and oral mucosa (open bars) were evaluated by real-time qPCR analysis. Data are mean (SEM). ${ }^{*} p<$ 0.05 and ${ }^{* *} p<0.01$ compared with radicular cysts.

3.3. Upregulated mRNA Expression of Transcription Factors (TEAD1, TEAD4, and RUNX2), Cell Cycle Related Genes (CDK2 and PCNA), YAP, and TAZ in KCOT. To investigate the expression levels of transcription factors and cell cycle related genes, the mRNA levels of TEAD1, TEAD4, RUNX2, CDK2, PCNA, YAP, and TAZ were explored in the samples of $\operatorname{KCOT~}(n=4)$ and normal mucosa $(n=3)$ by real-time qPCR analysis. The results showed that the tested genes were expressed in KCOT samples. The mRNA expressions of the TEAD1 $(p=0.0196)$, TEAD4 $(p=0.0351), \operatorname{RUNX} 2(p=$ $0.0015)$, CDK2 $(p=0.0209)$, PCNA $(p=0.0263)$, YAP $(p=0.0461)$, and TAZ $(p=0.0011)$ were significantly higher in samples from KCOT than those from OM (Figure 2).

3.4. Synchronous Distribution for YAP/TAZ and Proliferative Marker (Ki-67) in KCOT. To validate the correlation between YAP/TAZ activation and growth potential further, doublelabelling immunofluorescence analysis for YAP/TAZ and Ki67 was made on samples from KCOT and OM. As is shown in Figure 3, the characteristic YAP/TAZ dots were colocalised in the nucleus of the KCOT epithelium. The results showed that the YAP/TAZ was transported into the nucleus. There were also several colocalisations of YAP/TAZ with the Ki67 signals in the sections of KCOT (Figure 3, white arrow). However, immunofluorescent signals for YAP/TAZ or Ki-67 were rarely expressed in the epithelium of samples from oral mucosa.

3.5. Synchronous Distribution for Cyr61 and CTGF in KCOT and $O M$. To validate the correlation between downstream molecules of YAP/TAZ, double-labelling immunofluorescence analysis for Cyr61 and CTGF was made on samples from KCOT and OM. As is shown in Figure 4, the Cyr61 and CTGF signals are both present in the basal layers of KCOT and OM. Several overlapping expressions of Cyr61 and CTGF were detected in both samples of KCOT and OM (Figure 4, white arrow).

3.6. Correlation between Staining of YAP/TAZ, CTGF, Cyr61, and Ki-67 in KCOT. To further work out the potential significance of the activation of YAP/TAZ related to the proliferative
TABLE 1: Spearman rank analysis of the immunoreactivities. The data are given as $p$ values.

\begin{tabular}{lcccc}
\hline Marker & YAP/TAZ & Cyr61 & CTGF & Ki-67 \\
\hline YAP/TAZ & - & 0.0140 & 0.0250 & 0.0412 \\
Cyr61 & - & - & 0.1515 & 0.0291 \\
CTGF & - & - & - & 0.0003 \\
\hline
\end{tabular}

characteristics of KCOT, the relationship was then explored between the histoscores of YAP/TAZ, Cyr61, CTGF, and proliferative marker (Ki-67) in KCOT. The results showed significant correlation of the immunostaining of YAP/TAZ and Ki-67 (Table 1, $p=0.0412$ ). Besides, the immunostaining of YAP/TAZ downstream-related proteins (Cyr61, CTGF) was also shown to correlate with Ki-67 staining (Table $1, p=$ 0.0291, $p=0.0003$ ). All these findings suggested a possible link between activation of YAP/TAZ and growth potential in KCOT.

\section{Discussion}

First studied in Drosophila, YAP/TAZ was proved to play an important role in the control of organ size during development [13]. In several adult organs, YAP/TAZ not only play important roles for normal homeostasis but also are critical to promoting tissue repair upon injury [14-16]. Recently, accumulating evidences have supported that YAP and TAZ are oncogenes in various mammalian cells [17]. Genomewide analysis using mouse model of hepatocellular carcinomas showed chromosome amplification at 9qA1, which was syntenic to chromosome region 11q22 in human. YAP gene was in the amplified region and individually oncogenic [18]. YAP gene was also amplified in human cancers such as oral squamous cell carcinomas and medulloblastomas $[19,20]$. TAZ, also called WWTR1 (WW-domain containing transcriptional regulator 1), was first identified as a 14-3-3 binding protein in the year 2000 [21]. The human TAZ gene, located at 3q23-q24, encoded a $43 \mathrm{kDa}$ protein. TAZ was expressed in various tissues, but not thymus and peripheral blood leukocytes, and TAZ was also amplified in various human cancers such as breast cancers, non-small cell lung cancers, and gastric cancers [21].

Oral soft tissues were complicated biologic systems with the components responding to various physiologic stresses. These stresses included compression, hydrodynamic forces, elongation, friction, and shear stress generated during mastication, saliva flow, pronunciation, tooth-brushing, facial expression, and tooth eruption [22]. In the disease state such as jaw cysts, the cyst wall was exposed to hydrostatic pressures, osmotic pressures, and occlusal forces [23]. At cellular levels, cells could perceive external mechanical signals through various "mechanical sensors." These "mechanical sensors" include integrin based cell-matrix adhesions, stretch-modulated ion channels, and cell-cell junctions [24]. After sensing the mechanical cues, actin cytoskeleton could be activated, which is regulated by the RHO family of GTPases. Subsequently, contractile filamentous actin structures (F-actin) sustain YAP and TAZ nuclear localization and 


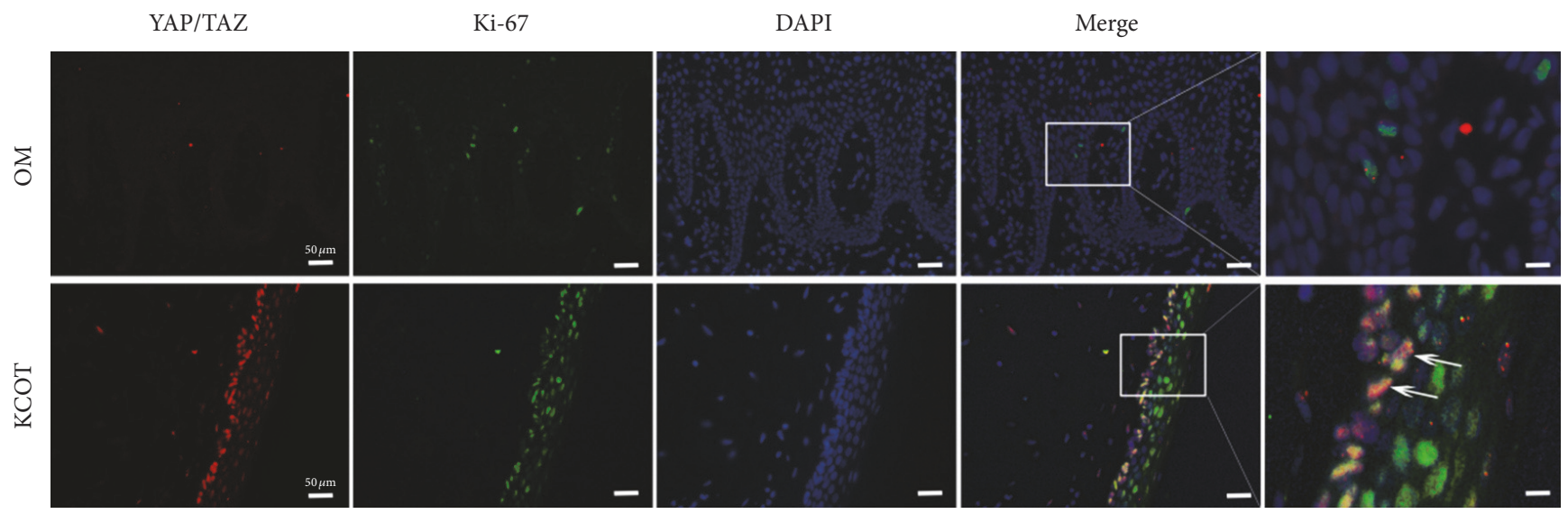

FIGURE 3: Double-labelling immunofluorescence staining for YAP/TAZ (red) and Ki-67 (green) in specimens of keratocystic odontogenic tumors and oral mucosa. The white arrow indicated the colocalisations of YAP/TAZ with Ki-67 signals in keratocystic odontogenic tumors.
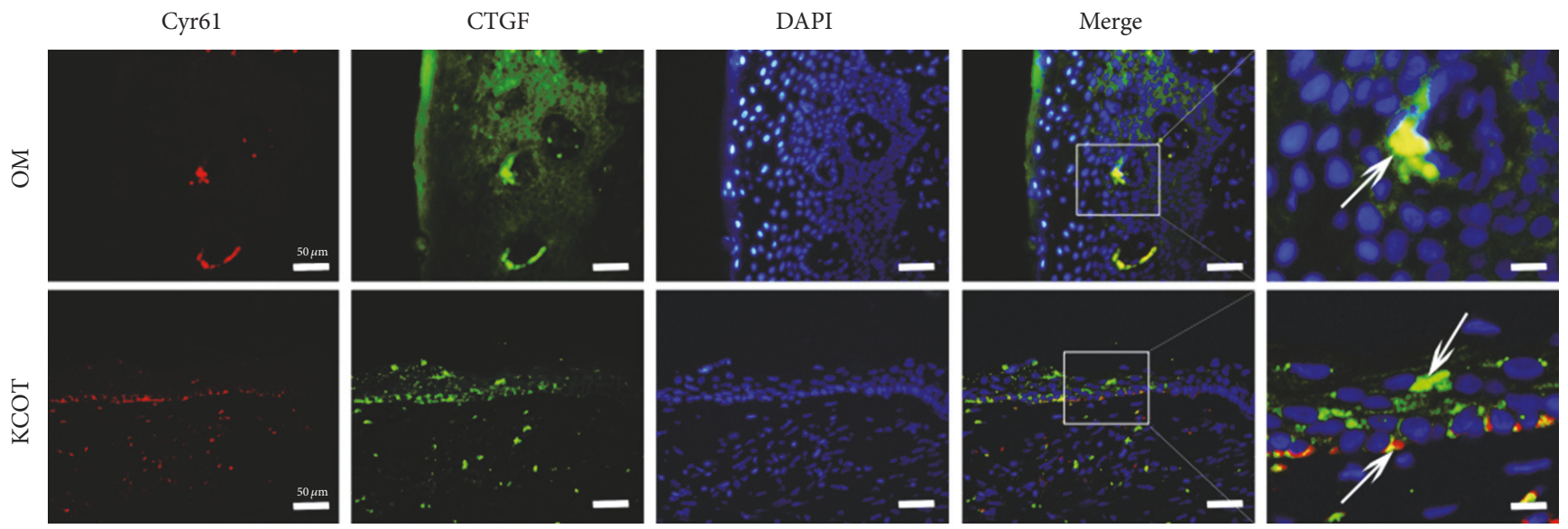

FIGURE 4: Double-labelling immunofluorescence staining for Cyr61 (red) and CTGF (green) in samples of keratocystic odontogenic tumors and oral mucosa. The white arrow indicated the colocalisations of Cyr61 and CTGF signals both in keratocystic odontogenic tumors and in oral mucosa.

activity [24]. YAP and TAZ had been shown to play important roles in organ development of oral region. For instance, $\alpha \mathrm{E}-$ catenin was an integral component of adherens junctions, where it linked cadherins to the actin cytoskeleton [25]. In the oral cavity, an elegant study has shown that $\alpha \mathrm{E}$-catenin restricts YAP/TAZ activity to establish a low proliferative cell group called enamel knot, which is a putative signaling center regulating tooth development [25]. Using YAP constitutive activation mice, other researchers also found that overexpression of YAP resulted in deformed tooth morphogenesis with widened dental lamina and mislocated enamel knot [26]. However, the expression status of YAP/TAZ in KCOT, a benign odontogenic tumor which arose from dental lamina and featured by mechanical loading, is still unclear $[1,4,23]$.

$\mathrm{YAP} / \mathrm{TAZ}$ dysregulation is wide spread in various human tumors, where YAP/TAZ have been shown to be indispensable for tumor initiation, progression, or metastasis [2731]. YAP/TAZ are transcriptional coactivators that shuttle between the cytoplasm and the nucleus, where they interact with other transcription factors, especially TEA domain family members (TEAD) [28]. YAP/TAZ/TEAD control the expression of their targets genes such as Cyr61 and CTGF [28]. Sustained nucleus activation of YAP/TAZ promotes aberrant cell proliferation and overcome anoikis [32]. In this study, the immunoreactivities of YAP/TAZ, Cyr61, CTGF, Ki67, YAP, and TAZ were significantly upregulated in KCOT. Besides, the mRNA level of the transcription factors such as TEAD1, TEAD4, RUNX2, YAP, and TAZ was also significantly upregulated in KCOT. All these findings suggest possible nucleus activation of YAP/TAZ in KCOT.

It has been widely known that KCOT owns a distinct growth behavior compared with the other kinds of jaw cysts and normal tissue [1]. The lining epithelium is proved to have a higher proliferative potential [33]. An emerging evidence is that aberrant expression of YAP/TAZ was possibly associated with the upregulated expression of tumor cell's proliferative markers [28]. In line with previous studies, our results also showed that the expression of Ki-67 was significantly higher in the epithelial layers than those in normal oral mucosa [34]. The significant relationship between the YAP/TAZ, Cyr61, 
CTGF, and Ki-67 was verified by Spearman rank correlation. The results from the double-labelling immunofluorescence also showed the partially synchronous distribution for YAP/TAZ and proliferative marker (Ki-67) in KCOT, which indicated the possible association between the YAP/TAZ activation and proliferative activity in KCOT.

\section{Conclusions}

In summary of the present study, the activation status of YAP/TAZ was investigated in the KCOT and also its possible association with aberrant proliferation characteristics of the KCOT epithelium was studied. As the activation status of YAP/TAZ may favor the unusual growth potential of KCOT, therefore, we have partially revealed the proliferative nature and the underlying mechanisms of KCOT. However, further investigation is still needed to uncover the precise molecular events behind the YAP/TAZ activation in KCOT.

\section{Ethical Approval}

The authors confirm that any tissue samples of the work covered in this manuscript have been conducted with the ethical approval of all relevant bodies and that such approvals are acknowledged within the manuscript.

\section{Conflicts of Interest}

The authors declare that they have no conflicts of interest.

\section{Acknowledgments}

The authors thank Professor Jia-Li Zhang for the help of histopathological check in identifying the KCOT. This study was supported by the Fundamental Research Funds for the Central Universities (121099) and National Natural Science Foundation of China (81300855).

\section{References}

[1] M. Shear, "The aggressive nature of the odontogenic keratocyst: is it a benign cystic neoplasm? Part 1. Clinical and early experimental evidence of aggressive behaviour," Oral Oncology, vol. 38, no. 3, pp. 219-226, 2002.

[2] L. D. R. Thompson, "World health organization classification of tumours: pathology and genetics of head and neck tumours," Ear, Nose and Throat Journal, vol. 85, no. 2, p. 74, 2006.

[3] H.-Y. Luo and T.-J. Li, "Odontogenic tumors: a study of 1309 cases in a Chinese population," Oral Oncology, vol. 45, no. 8, pp. 706-711, 2009.

[4] M. Shear, "The aggressive nature of the odontogenic keratocyst: is it a benign cystic neoplasm? Part 2. Proliferation and genetic studies," Oral Oncology, vol. 38, no. 4, pp. 323-331, 2002.

[5] N. Blanas, B. Freund, M. Schwartz, and I. M. Furst, "Systematic review of the treatment and prognosis of the odontogenic keratocyst," Oral Surgery, Oral Medicine, Oral Pathology, Oral Radiology, and Endodontics, vol. 90, no. 5, pp. 553-558, 2000.

[6] N. Kuroyanagi, H. Sakuma, S. Miyabe et al., "Prognostic factors for keratocystic odontogenic tumor (odontogenic keratocyst): analysis of clinico-pathologic and immunohistochemical findings in cysts treated by enucleation," Journal of Oral Pathology and Medicine, vol. 38, no. 4, pp. 386-392, 2009.

[7] A. Figueroa, M. Correnti, M. Avila, A. Andea, P. DeVilliers, and H. Rivera, "Keratocystic odontogenic tumor associated with nevoid basal cell carcinoma syndrome: similar behavior to sporadic type?" Otolaryngology-Head and Neck Surgery, vol. 142, no. 2, pp. 179-183, 2010.

[8] F. Zanconato, M. Cordenonsi, and S. Piccolo, "YAP/TAZ at the roots of cancer," Cancer Cell, vol. 29, no. 6, pp. 783-803, 2016.

[9] G. Halder and R. L. Johnson, "Hippo signaling: growth control and beyond," Development, vol. 138, no. 1, pp. 9-22, 2011.

[10] S. Dupont, L. Morsut, M. Aragona et al., "Role of YAP/TAZ in mechanotransduction," Nature, vol. 474, no. 7350, pp. 179-183, 2011.

[11] S. E. Hiemer, L. Zhang, V. K. Kartha et al., "A YAP/TAZregulated molecular signature is associated with Oral squamous cell carcinoma," Molecular Cancer Research, vol. 13, no. 6, pp. 957-968, 2015.

[12] R.-F. Li, G. Chen, Y. Zhao, Y.-F. Zhao, and B. Liu, "Increased expression of autophagy-related proteins in keratocystic odontogenic tumours: its possible association with growth potential," British Journal of Oral and Maxillofacial Surgery, vol. 52, no. 6, pp. 551-556, 2014.

[13] J. Huang, S. Wu, J. Barrera, K. Matthews, and D. Pan, “The Hippo signaling pathway coordinately regulates cell proliferation and apoptosis by inactivating Yorkie, the Drosophila homolog of YAP," Cell, vol. 122, no. 3, pp. 421-434, 2005.

[14] L. Azzolin, T. Panciera, S. Soligo et al., "YAP/TAZ incorporation in the $\beta$-catenin destruction complex orchestrates the Wnt response," Cell, vol. 158, no. 1, pp. 157-170, 2014.

[15] H. Bai, N. Zhang, Y. Xu et al., "Yes-associated protein regulates the hepatic response after bile duct ligation," Hepatology, vol. 56, no. 3, pp. 1097-1107, 2012.

[16] Q. Chen, N. Zhang, R. S. Gray et al., "A temporal requirement for Hippo signaling in mammary gland differentiation, growth, and tumorigenesis," Genes and Development, vol. 28, no. 5, pp. 432-437, 2014.

[17] B. Zhao, L. Li, Q. Lei, and K.-L. Guan, "The Hippo-YAP pathway in organ size control and tumorigenesis: an updated version," Genes and Development, vol. 24, no. 9, pp. 862-874, 2010.

[18] L. Zender, M. S. Spector, W. Xue et al., "Identification and validation of oncogenes in liver cancer using an integrative oncogenomic approach," Cell, vol. 125, no. 7, pp. 1253-1267, 2006.

[19] C. Baldwin, C. Garnis, L. Zhang, M. P. Rosin, and W. L. Lam, "Multiple microalterations detected at high frequency in oral cancer," Cancer Research, vol. 65, no. 17, pp. 7561-7567, 2005.

[20] A. Fernandez-L, P. A. Northcott, J. Dalton et al., "YAP1 is amplified and up-regulated in hedgehog-associated medulloblastomas and mediates Sonic hedgehog-driven neural precursor proliferation," Genes and Development, vol. 23, no. 23, pp. 27292741, 2009.

[21] F. Kanai, P. A. Marignani, D. Sarbassova et al., “TAZ: a novel transcriptional co-activator regulated by interactions with 143-3 and PDZ domain proteins," The EMBO Journal, vol. 19, no. 24, pp. 6778-6791, 2000.

[22] S. Goktas, J. J. Dmytryk, and P. S. McFetridge, "Biomechanical behavior of oral soft tissues," Journal of Periodontology, vol. 82, no. 8, pp. 1178-1186, 2011. 
[23] R. M. Browne, "The pathogenesis of odontogenic cysts: a review," Journal of Oral Pathology, vol. 4, no. 1, pp. 31-46, 1975.

[24] B. C. Low, C. Q. Pan, G. V. Shivashankar, A. Bershadsky, M. Sudol, and M. Sheetz, "YAP/TAZ as mechanosensors and mechanotransducers in regulating organ size and tumor growth," FEBS Letters, vol. 588, no. 16, pp. 2663-2670, 2014.

[25] C.-Y. Li, J. Hu, H. Lu et al., " $\alpha$ e-catenin inhibits YAP/TAZ activity to regulate signalling centre formation during tooth development," Nature Communications, vol. 7, Article ID 12133, 2016.

[26] M. Liu, S. Zhao, and X.-P. Wang, "YAP overexpression affects tooth morphogenesis and enamel knot patterning," Journal of Dental Research, vol. 93, no. 5, pp. 469-474, 2014.

[27] W. Jeong, S.-B. Kim, B. H. Sohn et al., "Activation of YAP1 is associated with poor prognosis and response to taxanes in ovarian cancer," Anticancer Research, vol. 34, no. 2, pp. 811-817, 2014.

[28] F. Zanconato, M. Forcato, G. Battilana et al., "Genome-wide association between YAP/TAZ/TEAD and AP-1 at enhancers drives oncogenic growth," Nature Cell Biology, vol. 17, no. 9, pp. 1218-1227, 2015.

[29] Z. Li, Y. Wang, Y. Zhu et al., "The Hippo transducer TAZ promotes epithelial to mesenchymal transition and cancer stem cell maintenance in oral cancer," Molecular Oncology, vol. 9, no. 6, pp. 1091-1105, 2015.

[30] Q. Wang, Z. Xu, Q. An et al., “TAZ promotes epithelial to mesenchymal transition via the upregulation of connective tissue growth factor expression in neuroblastoma cells," Molecular Medicine Reports, vol. 11, no. 2, pp. 982-988, 2015.

[31] T. Muramatsu, I. Imoto, T. Matsui et al., "YAP is a candidate oncogene for esophageal squamous cell carcinoma," Carcinogenesis, vol. 32, no. 3, pp. 389-398, 2011.

[32] M. Aragona, T. Panciera, A. Manfrin et al., "A mechanical checkpoint controls multicellular growth through YAP/TAZ regulation by actin-processing factors," Cell, vol. 154, no. 5, pp. 10471059, 2013.

[33] A. R. Gadbail, M. Chaudhary, S. Patil, and M. Gawande, "Actual Proliferating Index and p53 protein expression as prognostic marker in odontogenic cysts," Oral Diseases, vol. 15, no. 7, pp. 490-498, 2009.

[34] M. Shear, "The aggressive nature of the odontogenic keratocyst: is it a benign cystic neoplasm? Part 3. Immunocytochemistry of cytokeratin and other epithelial cell markers," Oral Oncology, vol. 38, no. 5, pp. 407-415, 2002. 


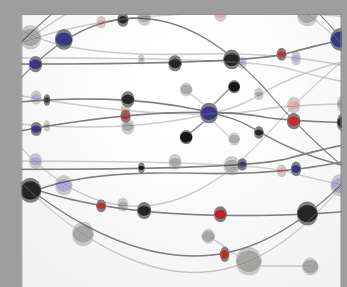

The Scientific World Journal
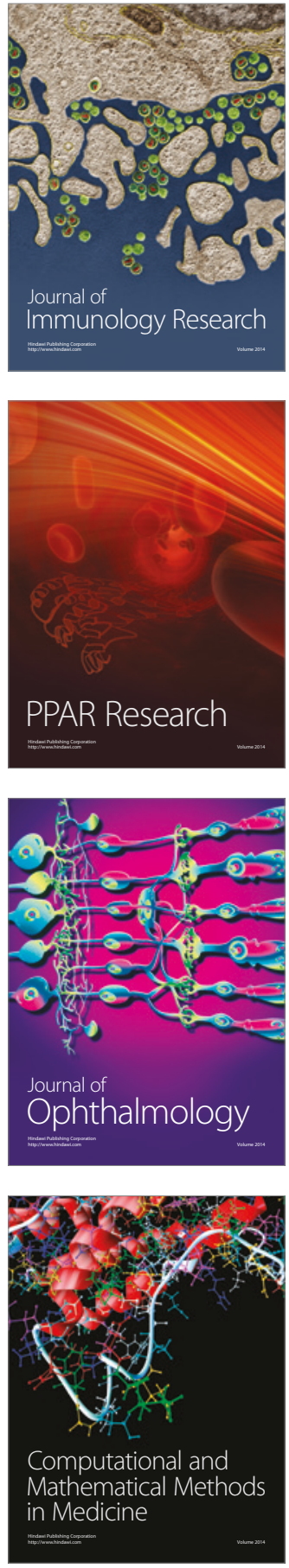

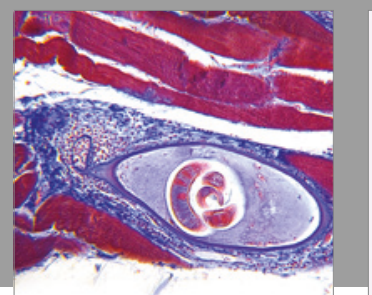

Gastroenterology Research and Practice
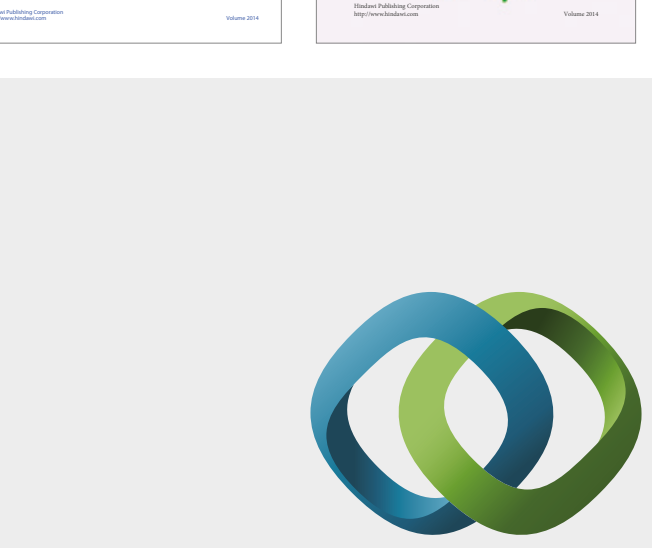

\section{Hindawi}

Submit your manuscripts at

https://www.hindawi.com
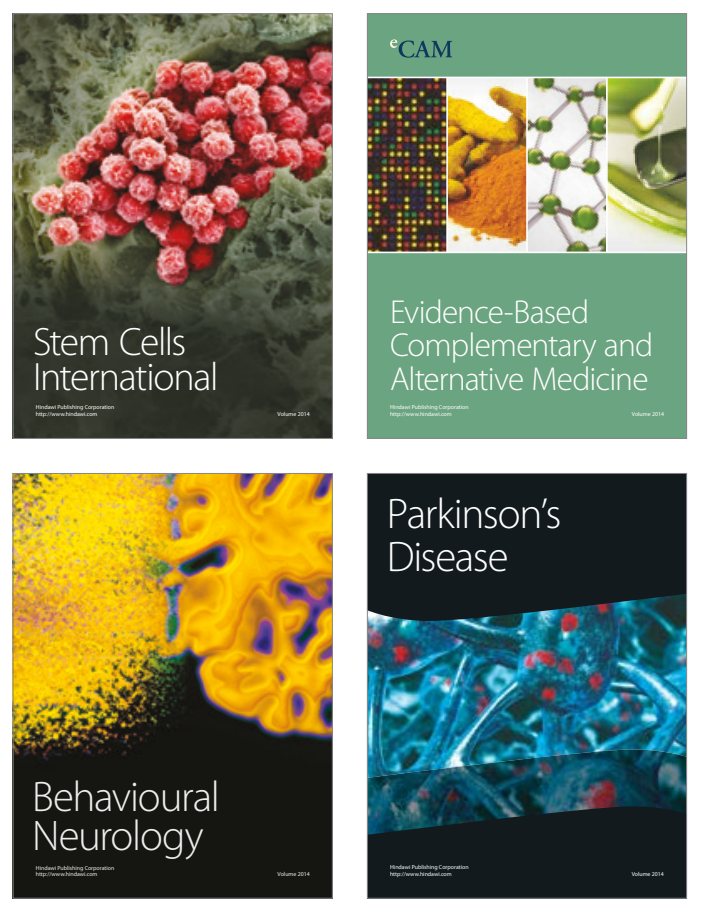
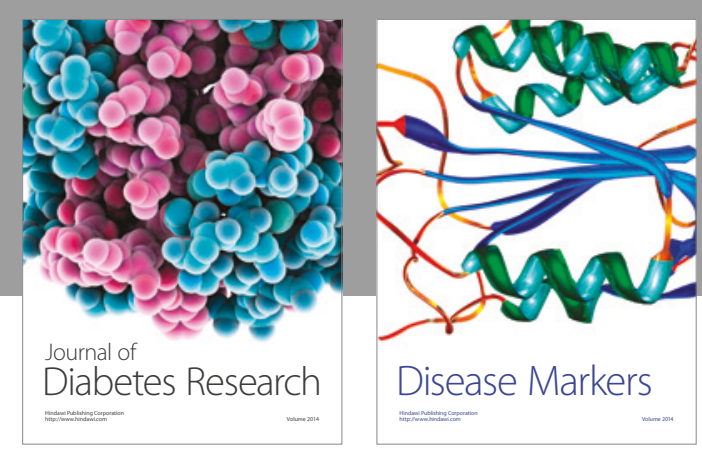

Disease Markers
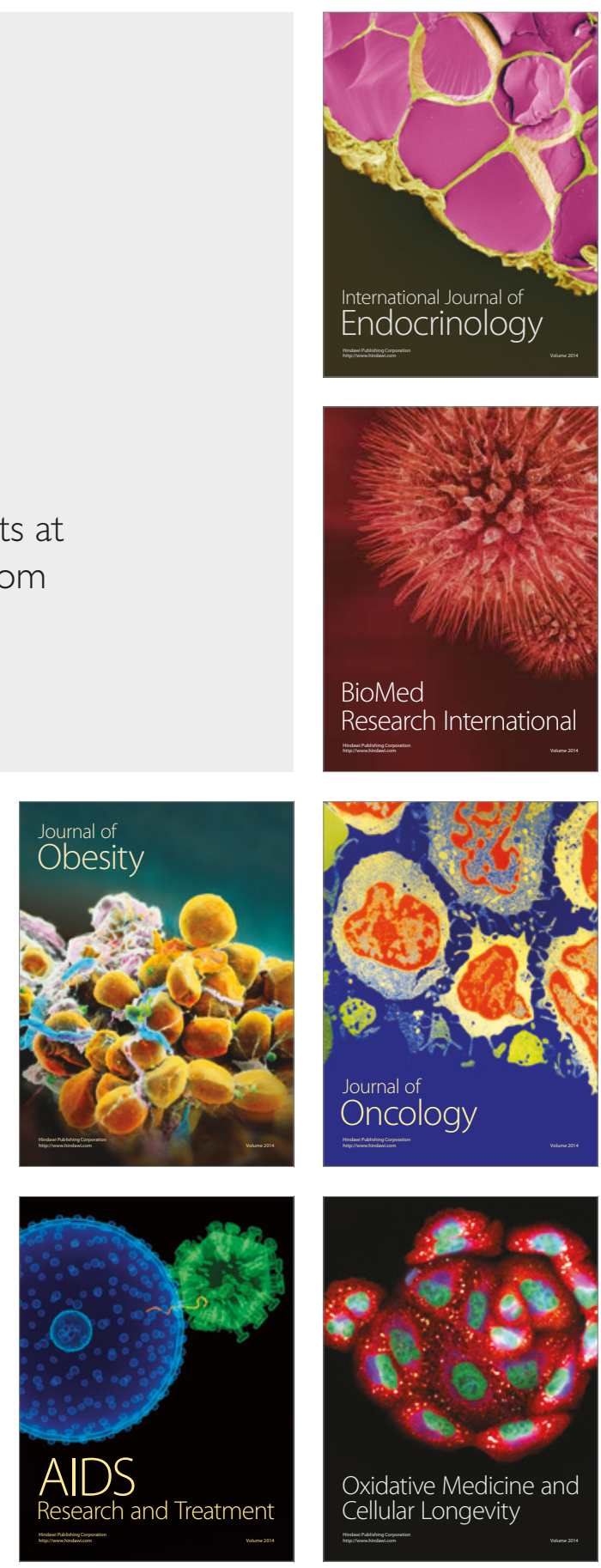